\title{
Jumps and induced anisotropy in the magnetoresistance of CuMn
}

\author{
S. Senoussi \\ Laboratoire de Physique des solides (*), Université Paris-Sud, 91405 Orsay, France \\ (Reçu le 22 septembre 1980, accepté le 2 décembre 1980)
}

\begin{abstract}
Résumé. - La magnétorésistance, $\Delta \rho / \rho_{0}$, de deux alliages CuMn de $4,7 \%$ et $1,35 \%$ a été mesurée dans des champs magnétiques $H$ compris entre + et $-35 \mathrm{kG}$ et dans le domaine de températures de l'hélium liquide. Lorsque l'aimantation rémanente saturée $\mathbf{m}_{\mathrm{r}}$ est parallèle au champ appliqué $\mathbf{H}$, supposé faible, $\Delta \rho / \rho_{0}$ varie linéairement en fonction de $H$ et présente des sauts brusques. Quand $\mathbf{H}$ est perpendiculaire à l'aimantation rémanente initiale la magnétorésistance varie comme $H^{2}$ mais elle est considérablement augmentée par rapport à la magnétorésistance réversible pour laquelle $m_{\mathrm{r}}=0$. Nous avons observé que la magnétorésistance ne dépend que de la somme vectorielle $\chi \mathbf{H}+\mathbf{m}_{\mathrm{r}}$ des aimantations réversibles et irréversibles macroscopiques.
\end{abstract}

\begin{abstract}
Magnetoresistances, $\Delta \rho / \rho_{0}$, of CuMn $4.7 \%$ and $1.35 \%$ have been measured in fields ranging between + and $-35 \mathrm{kG}$ and in the range of liquid helium temperatures. When the remanent magnetization $\mathbf{m}_{\mathrm{r}}$ is saturated and parallel to the field $\mathbf{H}, \Delta \rho / \rho_{0}$ varies linearly with $H$ for low fields and exhibits jumps. When the field is perpendicular to the initial remanence $m_{\mathrm{r}}, \Delta \rho / \rho_{0}$ is quadratic in $H$ but considerably enhanced with respect to the reversible magnetoresistance for which $m_{\mathrm{r}} \approx 0$. It is found that the magnetoresistance depends only on the vector sum $\chi \mathbf{H}+\mathbf{m}_{\mathbf{r}}$ of the macroscopic reversible and irreversible magnetizations.
\end{abstract}

1. Introduction. - Concentrated CuMn spin glass alloys have been known for a long time to exhibit a very narrow hysteresis cycle displaced towards negative fields [1, 2]. More recently, Monod et al. [3] have extended these works to much more dilute alloys $(600 \mathrm{ppm})$ and established that the magnetization reversal occurs in only one or a few discrete steps, implying a collective behaviour of the magnetization involved in the reversal. Using an N.M.R. technique, Alloul [4] reached the same conclusion from a microscopic point of view. The present author [5] has established by magnetoresistance measurements that the AuFe system, which has a very smooth symmetrical and featureless hysteresis loop, is homogeneous with no magnetic domains in the zero field cooled state on the scale of the electron mean free path $\lambda$ (homogeneous means that the microscopic properties of the alloys on a scale of the order of $\lambda$ are essentially identical to those of the macroscopic samples).

Magnetoresistance measurements on concentrated CuMn have been reported by several authors $[6,7,8]$. Schmitt and Jacobs were the first to measure the magnetoresistance of $\underline{\mathrm{CuMn}}$ spin glass alloys and they recognized the importance of the relation between $\Delta \rho$ and the square $m^{2}$ of the reversible magnetization.

(*) Laboratoire associé au C.N.R.S.
But all these studies concerned the reversible magnetoresistance while we are interested here in both the reversible and irreversible magnetoresistances as well as in their connections with the magnetization data. Among the magnetoresistance measurements on diluted CuMn alloys (few tens of ppm) we can mention the pioneering work of P. Monod [9] who established the existence of a negative term in the magnetoresistance of dilute $\underline{\mathrm{CuMn}}$ which varies as the square of the magnetization of the sample.

The aim of this letter is (I) to present the observation for the first time of jumps and remanent effects in the magnetotransport properties of $\mathrm{CuMn}$ spin glass, (II) to show that the magnetoresistance can, as for transverse susceptibility measurements, be considerably enhanced with respect to the purely reversible parallel magnetoresistance (for which the remanence is zero) when $H$ is perpendicular to the initial remanence $m_{r}$, (III) to show that the amplitude $\left|\mathbf{m}_{\mathbf{r}}\right|$ of the remanence remains constant (to within the irreversible decrease of $\mathbf{m}_{\mathrm{r}}$ [3]) while the magnetization reversal occurs, thus, excluding the other possible mechanisms where the amplitude $\left|\mathbf{m}_{\mathrm{r}}\right|$ of the remanence would go through zero as in the hypothesis of buckling or curling reversal [10].

Moreover preliminary analysis of the data indicates that, as for AuFe spin glass alloys [5], both the reversible $\chi H$, and the irreversible, $m_{\mathrm{r}}$ magnetizations are 
homogeneous on a scale of the order of $\lambda$ the electron mean free path (40 $\AA \approx \lambda \approx 140 \AA$ ). We recall that these conclusions are inferred from our finding [5] that the magnetoresistance (which probes the local correlations between the spins) and the magnetic measurements (which give the $z$ component $m_{z}$ of the macroscopic magnetization) provide almost same the value for $m$. It is for this reason that the magnetoresistance can be described in a homogeneous model such that :

$$
\begin{aligned}
\frac{\rho(H)-\rho_{0}}{\rho_{0}}=\frac{\Delta \rho(H)}{\rho_{0}} & =-\alpha m^{2} \\
& =-\alpha\left(\mathbf{m}_{\mathrm{r}}+\chi \mathbf{H}\right)^{2}
\end{aligned}
$$

(for low field)

where $\rho_{0}$ is the resistivity of the zero field cooled sample and $\rho(H)$ the resistivity in the field $H$. Although $\rho(H)$ appears to depend only on the total magnetization $\mathbf{m}$, it depends, as does $\mathbf{m}$, on all the magnetothermal history of the sample.

2. Experimental. - We have studied, using a standard A.C. four point contact technique, two CuMn samples with concentrations of 1.35 at. $\%$ and 4.7 at. $\%$. The samples in the form of thin plates were from the same alloys as those used for magnetization [3, 11], N.M.R. [11] and E.S.R. [12] studies. Fields up to $35 \mathrm{kG}$ could be applied and the sample direction could be rotated relative to the field. Because of the stray fields from the superconducting coil the origin of the field will be defined only to about $\pm 30 \mathrm{G}$. For the low field measurements $(|H| \approx 1 \mathrm{kG})$ the field sweep rate was about $1 \mathrm{kG} / \mathrm{min}$. The time constant of the measuring apparatus was $0.1 \mathrm{~s}$ and it was limited mainly by that of the lock-in amplifier (PAR $124 \mathrm{~A})$ which was used as a null detector at an excitation frequency equal to $22 \mathrm{~Hz}$. The noise $\delta$ represented a few ppm of the residual resistivity $\rho_{0}\left(\delta / \rho_{0} \simeq 3 \times 10^{-6}\right)$. It was checked, using a non magnetic sample ( 2.5 at. \%), that (1) the intrinsic hysteresis from the measuring apparatus was negligible (the superconducting magnet used had no superconducting switch or resistor in parallel), (2) the short time drift was about $2 \mathrm{ppm} / \mathrm{min}$. The form of the loop and the values of the low field magnetoresistance were found to be relatively insensitive to the field sweep rate ( $\sim 1 \mathrm{kG} / \mathrm{min}$.) as well as to the time constant of the set-up, but very dependent on the way that the remanent magnetization had been prepared. Hysteresis effects were observed even for fields as low as $1 \mathrm{kG}$. For the sake of clarity, we will neglect here such effects when they are small compared to the saturated remanence.

3. Results. - Figure 1 shows the low field magnetoresistances $\frac{\rho(H)-\rho_{0}}{\rho_{0}}$ versus $\mathbf{H}$ (for $|H| \approx 1 \mathrm{kG}$ ) of

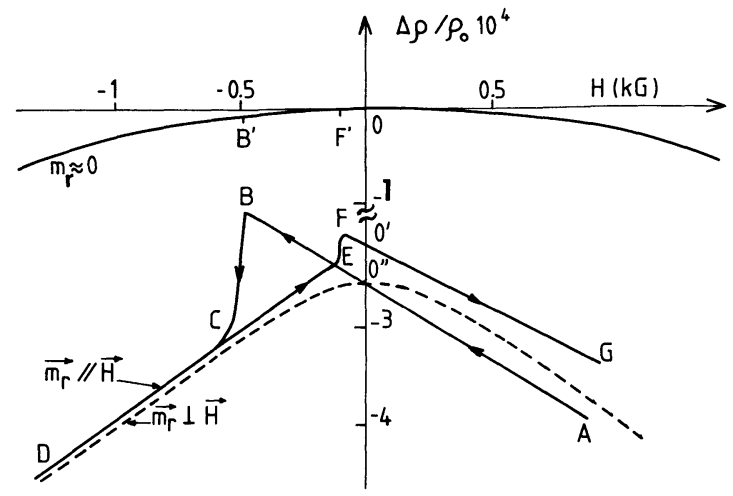

Fig. 1. - The low field magnetoresistance $\left(\Delta \rho / \rho_{0}\right.$ vs. H) of CuMn $4.7 \%$ at $4.2 \mathrm{~K}$ for three different states of the isothermal remanent magnetization $m_{\mathrm{r}}$. The upper curve corresponds to the zero field cooled sample $\left(m_{\mathrm{r}} \approx 0\right)$. In the case of the low and full loop, $m_{\mathrm{r}}$ is saturated and parallel to the applied field $\mathbf{H}$. For the dashed curve, $m_{\mathrm{r}}$ is also saturated but is now perpendicular to $\mathbf{H}$ (see the text for more details).

CuMn $4.7 \%$ at $4.2 \mathrm{~K}$ in three different magnetothermal conditions.

Upper curve (full line) was plotted following the sequence $(0 \rightarrow+1 \mathrm{kG} \rightarrow-1 \mathrm{kG} \rightarrow 0)$ after the sample had been cooled in zero field (Z.F.C.). Here the remanent effects, after completing the $\pm 1 \mathrm{kG}$ sweep, are quite small [13] and the magnetoresistance has the usual form appropriate to low fields

$$
\frac{\Delta \rho}{\rho_{0}}=-\beta H^{2}
$$

with $\beta(4.2)=(3.3 \pm 0.3) \cdot 10^{-5}(\mathrm{kG})^{-2}$.

In the case of the lower full loop, the Z.F.C. sample had been first submitted to a high positive field $\mathbf{H} / / \mathbf{z}(+35 \mathrm{kG})$ at $4.2 \mathrm{~K}$ resulting in a saturated isothermal remanent magnetization $\mathbf{m}_{\mathbf{r}}$ (I.R.M.). This high field was subsequently reduced down to about $+1 \mathrm{kG}$ (point A). The curve was then plotted, starting at $\mathrm{A}$, following the sequence A.B.C.D.E.F.G., or more precisely $+1 \mathrm{kG} \rightarrow-1 \mathrm{kG} \rightarrow+1 \mathrm{kG}$. Here the magnetoresistance exhibits a displaced cycle with two narrow jumps. The first one

$$
\left(\overline{\mathrm{BC}}=-(12.5 \pm 3) \cdot 10^{-5}\right)
$$

occurring at $H=H_{\mathrm{r}} \simeq-500 \mathrm{G}$ on the forward branch (A.B.C.D.), the other one

$$
\left(\overline{\mathrm{EF}}=(3.2 \pm 1) \cdot 10^{-5}\right)
$$

occurring at $H=H_{\mathrm{b}} \simeq-110 \mathrm{G}$ on the backward branch (D.E.F.G.). $H_{\mathrm{r}}$ and $H_{\mathrm{b}}$ are the corresponding reversal or coercive fields.

The comparison of the two full curves of figure 1 shows that the low field magnetoresistance of the sample in the saturated remanent magnetization state has lost any resemblance to the case where $m_{\mathrm{r}}=0$ (Z.F.C.). By contrast, the comparison with remanent magnetization loops from reference [3] shows striking similarity. Nevertheless, because of the fact that the 
jump width and the jump direction (up or down) are determined by the product $m_{\mathrm{r}} \chi H$ (this will be shown later), the cycle A.D.G. of figure 1 exhibits some typical and additional effects with respect to the magnetization loops. First the jump height $\overline{\mathrm{CB}}$ at $H_{\mathrm{r}}$ on the forward branch is in general much larger than the jump height $\overline{\mathrm{EF}}(\overline{\mathrm{CB}} / \overline{\mathrm{EF}} \simeq 4)$ at $H_{\mathrm{b}}$ on the backward branch. Secondly, the last segment (FG) of the backward branch is situated well above

$$
\left.\overline{\left(0^{\prime \prime} 0^{\prime}\right.} \simeq 4 \times 10^{-5}\right)
$$

the first segment $(\mathrm{AB})$ of the forward one. Besides, these two segments are not exactly parallel to each other. The slopes $\mathrm{d} / \mathrm{d} H\left(\Delta \rho / \rho_{0}\right)_{H=0}$ are equal to $-15 \times 10^{-5} \mathrm{kG}^{-1}$ at $0^{\prime \prime}$ and $-13 \times 10^{-5} \mathrm{kG}^{-1}$ at $0^{\prime}$. We now discuss the case of the dashed curve where the applied field, $H_{z}$, is perpendicular to the initial remanence $m_{\mathrm{r}}$ or to the anisotropy field

$$
\left(\mathbf{m}_{\mathrm{r}} / / \mathbf{H}_{\mathrm{A}} / / \mathbf{x}, \mathbf{H} / / \mathbf{z}\right) \text {. }
$$

Here the sample had been first submitted to a high field $H=H_{z}=35 \mathrm{kG}$, which was subsequently removed. The sample was then rotated through $90^{\circ}$ in zero field so that $m_{\mathrm{r}}$ was brought along the $\mathbf{x}$ direction. The dashed curve was then plotted following the sequence $(0 \rightarrow+1 \mathrm{kG} \rightarrow-1 \mathrm{kG} \rightarrow 0)$. As will be seen later, this magnetoresistance can be regarded as a measure of the transverse susceptibility of the sample [4]. Indeed, for low fields such as

$$
\left|H_{x}\right|<H_{\mathrm{A}} \simeq 500 \mathrm{G} \quad \text { [11] }
$$

the curve behaves very much like a parabola with an enhanced coefficient $\beta^{*} \approx 8.5 \beta \approx 26 \times 10^{-5}(\mathrm{kG})^{-2}$ where $\beta$ refers to the magnetoresistance of the Z.F.C. sample (upper curve and formula (3)). For $\left|H_{x}\right|>H_{\mathrm{A}}$ the magnetoresistance becomes more or less linear as a function of $H$ and almost superimposes the high field branches of the longitudinal loop where

$$
\mathbf{m}_{\mathbf{r}} / / \mathbf{H} \quad(|H| \approx 1 \mathrm{kG}) \text {. }
$$

We want to show now that all these various features associated with the curves of figure 1 can be explained, as was done for AuFe alloys [5], by using equations (1), (2) and some magnetic data from references [3] and [11].

Let us first consider the dashed curve in figure 1 for which $\mathbf{H} \perp \mathbf{H}_{\mathbf{A}}$. As was already established by Kouvel [1] and Alloul [4], for CuMn, the application of a transverse field $\mathbf{H} / / \mathbf{x}$ turns the remanent magnetization $\mathbf{m}_{\mathrm{r}}$ as a whole through an angle $\theta=(\mathbf{m}, \mathbf{z})$ which is determined by the equilibrium equation [11] (here we neglect the displacement field, see reference [11] for more details).

$$
\frac{\mathrm{d}}{\mathrm{d} \theta}\left(K \sin ^{2} \theta-m_{\mathrm{r}} H \sin \theta\right)=0 .
$$

If $\left|m_{\mathrm{r}} H / 2 K\right|<1$,

$$
\sin \theta=\frac{m_{\mathrm{r}} H}{2 K}=\frac{H}{H_{\mathrm{A}}}
$$

and

$$
\chi_{\mathrm{t}}=\chi\left(1+\frac{m_{\mathrm{r}}^{2}}{2 K \chi}\right)
$$

where $\chi_{\mathbf{t}}$ is the transverse susceptibility.

If $\left|m_{\mathrm{r}} H / 2 K\right|>1$,

$$
\sin \theta=1 \text {. }
$$

Therefore, for low fields equation (2) takes the form :

$$
\begin{aligned}
\frac{\Delta \rho}{\rho_{0}} & =-\alpha\left(\left(m_{\mathrm{r}} \cos \theta\right)^{2}+\left(m_{\mathrm{r}} \sin \theta+\chi H\right)^{2}\right) \\
& =-\alpha\left(m_{\mathrm{r}}^{2}+\chi^{2}\left(1+\frac{m_{\mathrm{r}}^{2}}{K \chi}\right) H^{2}\right) \\
& =-\alpha m_{\mathrm{r}}^{2}-\alpha\left(\chi^{*} H\right)^{2} .
\end{aligned}
$$

The term $-\alpha m_{\mathrm{r}}^{2}$ represents the reduction of the spin disorder resistivity of the sample arising from the presence of a remanence $m_{\mathrm{r}}$. For the $4.7 \% \mathrm{CuMn}$ sample one has $-\alpha m_{\mathrm{r}}^{2}=\overline{00}^{\prime \prime} \approx-2.6 \times 10^{-4}$ at $4.2 \mathrm{~K}$ (see Fig. 1). The second term

$$
-\alpha\left(\chi^{*} H\right)^{2}=-\beta^{*} H^{2}
$$

of equation (9) can be related to the transverse susceptibility $\chi^{*}$ or to the anisotropy field $H_{\mathrm{A}}$ :

$$
\begin{aligned}
\chi^{*}=\chi\left(1+\frac{m_{\mathrm{r}}^{2}}{K \chi}\right)^{1 / 2}=\chi(1 & \left.+2 \frac{m_{\mathrm{r}}}{\chi H_{\mathrm{A}}}\right)^{1 / 2}= \\
& =\chi\left(2 \frac{\chi_{\perp}}{\chi}-1\right)^{1 / 2} .
\end{aligned}
$$

The factor $1+\frac{m_{\mathrm{r}}^{2}}{K \chi}=1+\frac{2 m_{\mathrm{r}}}{\chi H_{\mathrm{A}}}=\eta=\beta^{*} / \beta$ can be defined as the enhancement factor of the magnetoresistance when $H \perp H_{\mathrm{A}}$ and for low field $\left(H<H_{\mathrm{A}}\right)$. For $\mathrm{CuMn} 4.7 \%$ we find $\eta(4.2 \mathrm{~K})=8.5 \pm 3$ and $\eta(1.2 \overline{\mathrm{K}})=38 \pm 8$ when $m_{\mathrm{r}}$ is saturated and at

$$
H \simeq 200 \mathrm{G} \text {. }
$$

These values compare satisfactorily with the quantities $\eta^{\prime}(4.2 \mathrm{~K})=8.7$ and $\eta^{\prime}(1.2 \mathrm{~K})=31$ as deduced from magnetic susceptibility data [14] at $H \approx 0$ $\left(\chi_{\perp} / \chi=4.8\right.$ and 16 at 4.2 and $1.2 \mathrm{~K}$ respectively) and equation (10) above.

We note that the comparison of our results with the zero field magnetic susceptibility data should be done only for low field values since we have found that for $H>300 \mathrm{G}$, such agreement fails. This, on the other hand, introduces relatively large uncertainties for the coefficient deduced from our data. In fact, we expect that for $H>H_{\mathrm{A}} \mathbf{m}_{\mathrm{r}}$ becomes parallel to and hence independent of $H$ (for $H$ not very high) so that equations (8), (9) and (10) are no longer valid while equation (2) takes the form

$$
\frac{\Delta \rho}{\rho_{0}}=-\alpha\left(m_{\mathrm{r}}^{2}+(\chi H)^{2}+2 m_{\mathrm{r}} \chi H\right)
$$


which is appropriate for describing both the dashed and the lower full curves for $H>H_{\mathrm{A}}$.

Let us apply now equation (2) to describe the curve of figure 1 where $H$ is parallel to the initial remanence. For the forward branch A.B.C.D. one has

$$
\begin{aligned}
& \Delta \rho / \rho_{0}=-\alpha\left(\chi H+m_{\mathrm{r}}\right)^{2} \quad \text { for } \quad H>H_{\mathrm{r}} \\
& \Delta \rho / \rho_{0}=-\alpha\left(\chi H-m_{\mathrm{r}}\right)^{2} \quad \text { for } \quad H<H_{\mathrm{r}} .
\end{aligned}
$$

The jump height is then equal to the discontinuity of $\Delta \rho / \rho_{0}$ at $H=H_{\mathrm{r}}$ :

$$
\overline{\mathrm{BC}}=J_{\mathrm{r}}=\frac{\rho\left(H_{\mathrm{r}}^{-}\right)-\rho\left(H_{\mathrm{r}}^{+}\right)}{\rho_{0}}=4 \alpha m_{\mathrm{r}} \chi H_{\mathrm{r}} .
$$

In the same way, the backward jump is given by

$$
\overline{\mathrm{EF}}=J_{\mathrm{b}}=\frac{\rho\left(H_{\mathrm{b}}^{+}\right)-\rho\left(H_{\mathrm{b}}^{-}\right)}{\rho_{0}}=-4 \alpha m_{\mathrm{r}} \chi H_{\mathrm{b}} .
$$

It is because the jump height is proportional to the reversal field that $J_{\mathrm{r}}$ and $J_{\mathrm{b}}$ differ in magnitudes and signs since $\left|H_{\mathrm{r}}\right| \gg\left|H_{\mathrm{b}}\right|$ in general. On the other hand $H_{\mathrm{r}}$ is always negative, while $H_{\mathrm{b}}$ may be positive or negative depending on the magnetic history of the sample. From equations (14) and (15) we obtain

$$
J_{\mathrm{r}} / J_{\mathrm{b}}=-H_{\mathrm{r}} / H_{\mathrm{b}} .
$$

Now from figure 1 we deduce

$$
J_{\mathrm{r}} / J_{\mathrm{b}}=\overline{\mathrm{BC}} / \overline{\mathrm{EF}} \simeq-4 \pm 0.4
$$

and $H_{\mathrm{r}} / H_{\mathrm{b}} \simeq 4.5$. These values are therefore, in good agreement with formula (16).

Finally we note that if $\left|m_{\mathrm{r}}\right|$ passes through zero during its reversal, the magnetoresistance would take the value

$$
\frac{\Delta \rho}{\rho_{0}}=-\alpha\left(\chi H_{\text {reversal }}\right)^{2} .
$$

Then the corresponding hysteresis loop would intersect the reversible curve (upper curve) at $\mathbf{B}^{\prime}$ and $\mathrm{F}^{\prime}$ since for this curve one has $\Delta \rho / \rho_{0}=-\alpha(\chi H)^{2}$. Therefore, one can conclude that during the jump, the amplitude $\left|m_{\mathrm{r}}\right|$ stays constant while $m_{\mathrm{r} z}$ passes through zero [3].

In the above analysis we have implicitly neglected any irreversible diminution in $m_{\mathrm{r}}$ during the time of the measurements. According to Monod et al. [3], in the best cases, the amplitude of the remanent magnetization amounts to only a reversal of $95 \%$ of the value it had just before the jump. An analysis of this effect in the magnetoresistance will be given elsewhere.

4. Conclusion. - We have shown that the magnetoresistance of CuMn spin glass, which is a measure of the microscopic correlations between Mn spins on the scale of $\lambda$ the conduction electron mean free path, agrees with the bulk magnetization data. This implies that, as in the case of AuFe [5], both the reversible and irreversible magnetizations of $\mathrm{CuMn}$ are homogeneously distributed through the sample on the scale of $\lambda$. In particular, our results show that if there are any magnetic domains in the Z.F.C. state these domains would have dimensions much smaller than $\lambda$ (probably less than $10 \AA$ for CuMn $4.7 \%$ for example). These conclusions thus confirm those already reached by Monod et al. [3] and Alloul [4] for CuMn.

In addition to the fact that the magnetoresistance measurements permit the study of the reversible and irreversible magnetization independently of each other through the terms $-\alpha(\chi H)^{2}$ (Z.F.C. sample) and $-\alpha m_{\mathrm{r}}^{2}$ (magnetoresistance in zero field) they also permit useful study of the microscopic coupling between these two quantities through the cross term $-\alpha m_{\mathrm{r}} \chi H$.

Finally we note that we have performed a systematic study of the evolution of the magnetoresistance loops of CuMn $4.7 \%$ and CuMn $1.35 \%$ under field or temperature cycling. Various effects on the remanent magnetization, the anisotropy field, and the displaced field have been observed and will be reported elsewhere. Also, with the collaboration of Guy [15] we have undertaken systematic magnetization and magnetoresistance measurements on identical CuMn, $\underline{\mathrm{AuFe}}, \underline{\mathrm{AuMn}}$ and $\underline{\mathrm{AuCr}}$ alloys.

Acknowledgments. - I wish to thank Dr. I. A. Campbell and Dr. P. Monod for many fruitful discussions and critical reading of the manuscript. I should also like to thank Dr. C. Guy for many stimulating discussions and F. Hippert for communication of some magnetization data. 


\section{References}

[1] Kouvel, J. S., J. Phys. Chem. Solids 21 (1961) 57.

[2] KondorskiI, E. I., Galkina, O. S., IvanovskiI, V. I., TCheRemushinina, A. V., Usarov, U. T., DJuvakulov, A., Ivanov-SmolenskiI, G. A. and Puchkova, S. M., Proc. of the Int. Conf. Mag. I.C.M. 734528 Moscow.

[3] Monod, P., Prejean, J. J. and Tissier, B., J. Appl. Phys. 50 (1979) 7324.

[4] Alloul, H., Phys. Rev. Lett. 42 (1979) 603; J. Appl. Phys. 50 (1979) 7330.

[5] SenoussI, S., to be published in J. Phys. F, Metal Physics (1980).

[6] Schmitt, R. W. and JACoBs, I. S., J. Phys. Chem. Solids 3 (1957) 324.
[7] Hurd, C. M. and Alderson, J. E., Phys. Rev. B 4 (1971) 1088.

[8] McAlister, S. P. and Hurd, C. M., Phys. Rev. B 20 (1979) 1113.

[9] Monod, P., Phys. Rev. Lett. 79 (1967) 1113.

[10] Frei, E. H., Shtrikman, S. and Trevers, D., Phys. Rev. 106 (1957) 446

[11] Alloul, H. and Hippert, F., J. Physique Lett. 41 (1980) L-201.

[12] MONOD, P. and BeRTHIER, Y., I.C.M. 79 Munich J.M.M.M. (1980).

[13] Careaga, A., Thesis, Grenoble (1967), unpublished.

[14] HIPPERT, F., private communications.

[15] GuY, C. and Senoussi, S., to be published. 